MATHEMATICS OF COMPUTATION

Volume 75, Number 256, October 2006, Pages 1987-1995

S 0025-5718(06)01856-4

Article electronically published on May 16, 2006

\title{
LOWER BOUNDS FOR THE CONDITION NUMBER OF A REAL CONFLUENT VANDERMONDE MATRIX
}

\author{
REN-CANG LI
}

\begin{abstract}
Lower bounds on the condition number $\kappa_{p}\left(V_{\mathrm{c}}\right)$ of a real confluent Vandermonde matrix $V_{\mathrm{c}}$ are established in terms of the dimension $n$, or $n$ and the largest absolute value among all nodes that define the confluent Vandermonde matrix and the interval that contains the nodes. In particular, it is proved that for any modest $k_{\max }$ (the largest multiplicity of distinct nodes), $\kappa_{p}\left(V_{\mathrm{c}}\right)$ behaves no smaller than $\mathcal{O}_{n}\left((1+\sqrt{2})^{n}\right)$, or than $\mathcal{O}_{n}\left((1+\sqrt{2})^{2 n}\right)$ if all nodes are nonnegative. It is not clear whether those bounds are asymptotically sharp for modest $k_{\max }$.
\end{abstract}

\section{INTRODUCTION}

Given $n$ numbers $\alpha_{1}, \alpha_{2}, \ldots, \alpha_{n}$ called nodes, the associated Vandermonde matrix is defined as

$$
V \stackrel{\text { def }}{=}\left(\begin{array}{cccc}
1 & 1 & \cdots & 1 \\
\alpha_{1} & \alpha_{2} & \cdots & \alpha_{n} \\
\vdots & \vdots & \ddots & \vdots \\
\alpha_{1}^{n-1} & \alpha_{2}^{n-1} & \cdots & \alpha_{n}^{n-1}
\end{array}\right) .
$$

It, for example, arises from polynomial interpolation and others 3.6 is invertible if all nodes $\alpha_{j}$ are distinct, i.e., $\alpha_{i} \neq \alpha_{j}$ for $i \neq j$, but it becomes singular whenever $\alpha_{i}=\alpha_{j}$ for some $i \neq j$. A generalization of $V$ for nodes not all of which are distinct is the so-called confluent Vandermonde matrices, e.g.,

$$
\left(\begin{array}{cccccc}
1 & 0 & 0 & 1 & 1 & 0 \\
\alpha_{1} & 1 & 0 & \alpha_{4} & \alpha_{5} & 1 \\
\alpha_{1}^{2} & 2 \alpha_{1} & 2 & \alpha_{4}^{2} & \alpha_{5}^{2} & 2 \alpha_{5} \\
\alpha_{1}^{3} & 3 \alpha_{1}^{2} & 6 \alpha_{1} & \alpha_{4}^{3} & \alpha_{5}^{3} & 3 \alpha_{5}^{2} \\
\alpha_{1}^{4} & 4 \alpha_{1}^{3} & 12 \alpha_{1}^{2} & \alpha_{4}^{4} & \alpha_{5}^{4} & 4 \alpha_{5}^{3} \\
\alpha_{1}^{5} & 5 \alpha_{1}^{4} & 20 \alpha_{1}^{3} & \alpha_{4}^{5} & \alpha_{5}^{5} & 5 \alpha_{5}^{4}
\end{array}\right)
$$

where $\alpha_{1}=\alpha_{2}=\alpha_{3}$ and $\alpha_{5}=\alpha_{6}$. The second, third, and sixth columns are obtained by "differentiating" the previous column. Confluent Vandermonde matrices

Received by the editor October 20, 2004 and, in revised form, May 23, 2005.

2000 Mathematics Subject Classification. Primary 15A12, 65F35.

Key words and phrases. Optimal condition number, Vandermonde matrix, confluent Vandermonde matrix, Chebyshev polynomials.

This work was supported in part by the National Science Foundation CAREER award under Grant No. CCR-9875201 and by the National Science Foundation under Grant No. DMS-0510664. 
arise in Hermite interpolation [4, for example. Adopting the formulation in [8], we define the confluent Vandermonde matrix $V_{\mathrm{c}}$ as follows. First

$$
\begin{aligned}
& \left\{\alpha_{j}\right\}_{j=1}^{n} \text { are ordered so that equal nodes are contiguous, i.e., } \\
& \qquad \alpha_{i}=\alpha_{j} \quad(i<j) \quad \Rightarrow \quad \alpha_{i}=\alpha_{i+1}=\cdots=\alpha_{j} .
\end{aligned}
$$

Define

$$
V_{\mathrm{c}}=\left(f_{1}\left(\alpha_{1}\right) f_{2}\left(\alpha_{2}\right) \cdots f_{n}\left(\alpha_{n}\right)\right),
$$

where the vector function $f_{j}(t)$ is defined recursively by

$$
f_{j}(t)= \begin{cases}\left(1 t \cdots t^{n-1}\right)^{T}, & \text { if } j=1 \text { or } \alpha_{j} \neq \alpha_{j-1}, \\ \frac{d}{d x} f_{j-1}(t), & \text { otherwise }\end{cases}
$$

where ".T" is the transpose of a vector or matrix. As far as defining $V_{\mathrm{c}}$ is concerned, $\alpha_{j}$ can be real or complex. But in this paper, we shall focus on real $\alpha_{j}$. In what follows, $\alpha_{j}$ and $V_{\mathrm{c}}$, as well as

$$
\alpha_{\max } \stackrel{\text { def }}{=} \max _{j}\left|\alpha_{j}\right|
$$

are reserved for their assignments here.

(Optimal) condition numbers for real Vandermonde matrices have been systematically studied by Gautschi and his coauthor (see [7] and references therein), and more recently by Tyrtyshnikov [12, Beckermann 2], and Li [10. In this paper, we shall establish three lower bounds on the $\ell_{p}$-condition number $\kappa_{p}\left(V_{\mathrm{c}}\right) \equiv$ $\left\|V_{\mathrm{c}}\right\|_{p}\left\|V_{\mathrm{c}}^{-1}\right\|_{p}$ in terms of $n$, or $n$ and $\alpha_{\max }$ and the interval $[\alpha, \beta]$ that contains all nodes. In particular, we will show that for fixed $k_{\max }$ (the largest multiplicity of distinct nodes), $\kappa_{p}\left(V_{\mathrm{c}}\right)$ behaves no smaller than $\mathcal{O}_{n}\left((1+\sqrt{2})^{n}\right)$, where notation $a_{n}=\mathcal{O}_{n}\left(b_{n}\right)$ means $c_{1} n^{d_{1}} \leq a_{n} / b_{n} \leq c_{2} n^{d_{2}}$ for some constants $c_{1}, c_{2}, d_{1}$, and $d_{2}$.

Optimally conditioned confluent Vandermonde matrices can be much worse illconditioned than optimally conditioned Vandermonde matrices. One extreme example would be that all nodes are equal $\alpha_{1}=\cdots=\alpha_{n}$ for which $V_{\mathrm{c}}$ is lower triangular, and thus

$$
\kappa_{p}\left(V_{\mathrm{c}}\right) \geq(n-1) ! \sim \sqrt{2 \pi} n^{n-1 / 2} e^{-n}
$$

by Stirling's asymptotic formula [1, Page 18], and it becomes an equality for $\alpha_{1}=$ $\cdots=\alpha_{n}=0$. While for optimally conditioned Vandermonde matrices, $\kappa_{p}(V)$ goes to $\infty$ as fast as $(1+\sqrt{2})^{n}$ modulo a factor $n^{d}$ for $|d| \leq 1$ [2, 10].

The rest of this paper is organized as follows. A general lower bound on $\kappa_{p}\left(V_{\mathrm{c}}\right)$ is established in Section 2, but it is not uniform. Uniform bounds for $p=\infty$ are obtained in Section 3 for all real $V_{\mathrm{c}}$ and for $V_{\mathrm{c}}$ with nonnegative nodes. Finally we present our concluding remarks in Section 4

\section{A general LOWER BOUND}

Given $1 \leq p \leq \infty$, the $\ell_{p}$-norm of vector $u=\left(\begin{array}{lll}\mu_{1} & \mu_{2} \cdots \mu_{n}\end{array}\right)^{T}$ is defined as

$$
\|u\|_{p}=\left(\sum_{j=1}^{n}\left|\mu_{j}\right|^{p}\right)^{1 / p}
$$


and $\|u\|_{\infty}=\lim _{p \rightarrow \infty}\|u\|_{p}=\max _{j}\left|\mu_{j}\right|$. The associated $\ell_{p}$-operator norm of the $m \times n$ matrix $A$ is defined as

$$
\|A\|_{p}=\max _{u \neq 0} \frac{\|A u\|_{p}}{\|u\|_{p}} .
$$

It can be proved that $\|A\|_{p}=\left\|A^{T}\right\|_{p^{\prime}}$, upon noticing

$$
\|A\|_{p}=\max _{u \neq 0, v \neq 0} \frac{\left|v^{T} A u\right|}{\|v\|_{p^{\prime}}\|u\|_{p}},
$$

where $1 / p+1 / p^{\prime}=1$ (see also 9 ).

Let $[\alpha, \beta]$ be the interval in which all $\alpha_{j}$ lie.

$$
\begin{aligned}
T_{n}(t) & =\cos (n \arccos t) & & \text { for }|t| \leq 1, \\
& =\frac{1}{2}\left(t+\sqrt{t^{2}-1}\right)^{n}+\frac{1}{2}\left(t-\sqrt{t^{2}-1}\right)^{n} & & \text { for }|t| \geq 1
\end{aligned}
$$

is the $n$th Chebyshev polynomial of the first kind. Define the $n$th translated Chebyshev polynomial $T_{n}(x ; \omega, \tau) \stackrel{\text { def }}{=} T_{n}(x / \omega+\tau)$, where

$$
\omega=\frac{\beta-\alpha}{2}>0, \quad \tau=-\frac{\beta+\alpha}{\beta-\alpha} .
$$

Let $a_{j n} \equiv a_{j n}(\omega, \tau)$ be the coefficient of $x^{j}$ in $T_{n}(x ; \omega, \tau)$, i.e.,

$$
T_{n}(x ; \omega, \tau)=a_{n n} x^{n}+a_{n-1 n} x^{n-1}+\cdots+a_{1 n} x+a_{0 n} .
$$

Define [10]

$$
S_{n, p}(\omega, \tau)=\left(\sum_{j=0}^{n}\left|a_{j n}\right|^{p}\right)^{1 / p} .
$$

Now we are ready to state our main theorem for the section.

Theorem 2.1. Assume that there are $\ell$ distinct nodes $\alpha_{j}$, and let $k_{\max }$ be the largest multiplicity of the distinct nodes. Then

$$
\kappa_{p}\left(V_{\mathrm{c}}\right) \geq \min _{1 \leq k \leq k_{\max }}\left[\frac{(n-k) !}{(n-1) !}\right]^{2} \omega^{k-1} \times \max \left\{\ell^{1 / p^{\prime}}, \alpha_{\max }^{n-1}\right\} \frac{S_{n-1, p^{\prime}}(\omega, \tau)}{n^{1 / p^{\prime}}} .
$$

Proof. Inequality (2.5) is a consequence of Lemmas 2.1 and 2.3 below.

For $k_{\max }=1$, i.e., $\ell=n$ and $k_{1}=\cdots=k_{n}=1$ (and thus $V_{\mathrm{c}}=V$ ), (2.5) becomes one of the lower bounds for $\kappa_{p}(V)$ in [10. The right-hand side of (2.5) entails the explicit computation of $S_{n, p^{\prime}}(\omega, \tau)$. It can also be estimated fairly well, too, by

$$
\begin{aligned}
n^{-1 / p} S_{n-1,1}(\omega, \tau) & \leq S_{n-1, p^{\prime}}(\omega, \tau) \leq S_{n-1,1}(\omega, \tau), \\
\lceil n / 2\rceil^{-1 / p} S_{n-1,1}(\omega, 0) \leq & S_{n-1, p^{\prime}}(\omega, 0) \leq S_{n-1,1}(\omega, 0),
\end{aligned}
$$

in connection with the explicit formulas for $S_{n-1,1}(\omega, \tau)$ for $\tau=0$ or $|\tau| \geq 1$ in [10]. Here $\lceil\xi\rceil$ is the smallest integer that is larger than $\xi$. The formulas are

$$
S_{n-1,1}(\omega, 0)=T_{n-1}(\iota / \omega) \sim \frac{1}{2}\left(\frac{1}{\omega}+\sqrt{1+\frac{1}{\omega^{2}}}\right)^{n-1},
$$


where $\iota=\sqrt{-1}$, and for $\alpha \geq 0$ (for which $\tau \leq-1$ ),

$$
S_{n-1,1}(\omega, \tau)=T_{n-1}(|\tau|+1 / \omega) \sim \frac{1}{2}\left[\left(\frac{1}{\omega}+|\tau|\right)+\sqrt{\left(\frac{1}{\omega}+|\tau|\right)^{2}-1}\right]^{n-1}
$$

Lemma 2.1. Assume that there are $\ell$ distinct nodes $\alpha_{j}$. Then

$$
\begin{aligned}
& \left\|V_{\mathrm{c}}\right\|_{p} \geq \max \left\{\ell^{1 / p^{\prime}}, \alpha_{\max }^{n-1}\right\}, \\
& \left\|V_{\mathrm{c}}\right\|_{p} \geq\left(\sum_{j=1}^{n} \alpha_{\max }^{(j-1) p}\right)^{1 / p} .
\end{aligned}
$$

Proof. Let $e_{j}$ be the $j$ th column of the $n \times n$ identity matrix $I_{n}$ (or simply $I$ if $n$ is clear from the context). Use $\left\|V_{\mathrm{c}}\right\|_{p} \geq\left\|V_{\mathrm{c}}^{T} e_{1}\right\|_{p^{\prime}}$ and $\left\|V_{\mathrm{c}}\right\|_{p} \geq\left\|V_{\mathrm{c}}^{T} e_{n}\right\|_{p^{\prime}}$ to get (2.10), and use $\left\|V_{\mathrm{c}}\right\|_{p} \geq \max _{j}\left\|V_{\mathrm{c}}^{T} e_{j}\right\|_{p}$ to get (2.11).

Lemma 2.2. For $0 \leq k \leq n$,

$$
\left|\frac{d}{d x^{k}} T_{n}(x ; \omega, \tau)\right| \leq \frac{[n(n-1) \cdots(n-k+1)]^{2}}{\omega^{k}} \quad \text { for } x \in[\alpha, \beta] .
$$

Proof. It follows from $T_{n}(x ; \omega, \tau)=T_{n}(x / \omega+\tau) \equiv T_{n}(t)$ that

$$
\frac{d^{k}}{d x^{k}} T_{n}(x ; \omega, \tau)=\frac{1}{\omega^{k}} T_{n}^{(k)}(t),
$$

where $t \equiv t(x)=x / \omega+\tau$. It suffices to show that $\left|T_{n}^{(k)}(t)\right| \leq[n(n-1) \cdots(n-k+1)]^{2}$ for $t \in[-1,1]$ since $t(x)$ maps $x \in[\alpha, \beta]$ to $t \in[-1,1]$. By Markov's inequality [5, Page 233],

$$
\begin{aligned}
\max _{t \in[-1,1]}\left|T_{n}^{(k)}(t)\right| & \leq(n-k+1)^{2} \max _{t \in[-1,1]}\left|T_{n}^{(k-1)}(t)\right| \\
& \leq \cdots \\
& \leq[n(n-1) \cdots(n-k+1)]^{2} \max _{t \in[-1,1]}\left|T_{n}(t)\right| \\
& =[n(n-1) \cdots(n-k+1)]^{2},
\end{aligned}
$$

as expected.

Lemma 2.3. Under the conditions of Theorem 2.1,

$$
\left\|V_{\mathrm{c}}^{-1}\right\|_{p} \geq \min _{1 \leq k \leq k_{\max }}\left[\frac{(n-k) !}{(n-1) !}\right]^{2} \omega^{k-1} \times \frac{S_{n-1, p^{\prime}}(\omega, \tau)}{n^{1 / p^{\prime}}} .
$$

Proof. For the sake of this proof, let the $\ell$ distinct nodes have multiplicities $k_{1}, k_{2}, \ldots, k_{\ell}$, respectively, where $k_{1}+k_{2}+\cdots+k_{\ell}=n$, and the first $k_{1} \alpha_{j}$ 's are equal, the next $k_{2} \alpha_{j}$ 's are equal, and so on. Let $v$ be the vector of the coefficients of the translated Chebyshev polynomial $T_{n-1}(x ; \omega, \tau)$, i.e., $v=$ $\left(\begin{array}{llll}a_{0 n-1} & a_{1 n-1} & \cdots & a_{n-1 n-1}\end{array}\right)^{T}$. Then

$$
V_{\mathrm{c}}^{T} v=\left(T_{n-1}\left(\alpha_{1} ; \omega, \tau\right) T_{n-1}^{\prime}\left(\alpha_{1} ; \omega, \tau\right) \cdots T_{n-1}^{\left(k_{1}-1\right)}\left(\alpha_{1} ; \omega, \tau\right) \cdots \cdots\right)^{T},
$$


which yields, by Lemma 2.2 for $1 \leq p^{\prime}<\infty$

$$
\begin{aligned}
\left\|V_{\mathrm{c}}^{T} v\right\|_{p^{\prime}}^{p^{\prime} \leq} & \sum_{j=1}^{\ell}\left(1^{p^{\prime}}+\left[\frac{(n-1)^{2}}{\omega}\right]^{p^{\prime}}\right. \\
& \left.+\cdots+\left[\frac{\left[(n-1)(n-2) \cdots\left(n-k_{j}+1\right)\right]^{2}}{\omega^{k_{j}-1}}\right]^{p^{\prime}}\right) \\
\leq & \sum_{j=1}^{\ell} k_{j} \times\left(\max _{1 \leq k \leq k_{j}}\left[\frac{(n-1) !}{(n-k) !}\right]^{2} \frac{1}{\omega^{k-1}}\right)^{p^{\prime}} \\
\leq & n \times\left(\max _{1 \leq k \leq k_{\max }}\left[\frac{(n-1) !}{(n-k) !}\right]^{2} \frac{1}{\omega^{k-1}}\right)^{p^{\prime}},
\end{aligned}
$$

which gives

$$
\left\|V_{\mathrm{c}}^{T} v\right\|_{p^{\prime}} \leq n^{1 / p^{\prime}} \times \max _{1 \leq k \leq k_{\max }}\left[\frac{(n-1) !}{(n-k) !}\right]^{2} \frac{1}{\omega^{k-1}} .
$$

This is proved so far for $1 \leq p^{\prime}<\infty$, but it can be verified that (2.16) holds for $p^{\prime}=\infty$, too. Therefore, we have

$$
\begin{aligned}
\left\|V_{\mathrm{c}}^{-T}\right\|_{p^{\prime}} & =\max _{u} \frac{\|u\|_{p^{\prime}}}{\left\|V_{\mathrm{c}}^{T} u\right\|_{p^{\prime}}} \geq \frac{\|v\|_{p^{\prime}}}{\left\|V_{\mathrm{c}}^{T} v\right\|_{p^{\prime}}} \\
& \geq \min _{1 \leq k \leq k_{\max }}\left[\frac{(n-k) !}{(n-1) !}\right]^{2} \omega^{k-1} \times \frac{S_{n-1, p^{\prime}}(\omega, \tau)}{n^{1 / p^{\prime}}},
\end{aligned}
$$

as was to be shown.

In general, we may use (2.14), instead of (2.15), in estimating $\left\|V_{\mathrm{c}}^{-1}\right\|_{p}$. Doing so, however, will lead to a more complicated lower bound on $\kappa_{p}\left(V_{\mathrm{c}}\right)$.

Remark 2.1. Lemma 2.3 is made possible by Lemma 2.2 which is proved with the help of Markov's inequality. Another classical inequality for the same purpose is Bernstein's inequality [5. Page 233], using which we can obtain the following. For $0 \leq k \leq n$, if $\alpha<a \stackrel{\text { def }}{=} \min _{j} \alpha_{j}<b \stackrel{\text { def }}{=} \max _{j} \alpha_{j}<\beta$, then

$$
\left|\frac{d}{d x^{k}} T_{n}(x ; \omega, \tau)\right| \leq \frac{n(n-1) \cdots(n-k+1)}{\left[\omega \sqrt{1-\left(\frac{\max \{\beta-b, a-\alpha\}}{\omega}\right)^{2}}\right]^{k}} \text { for } x \in[\alpha, \beta] .
$$

This inequality improves (2.12) in the numerator part but has complications in the denominator, and also it requires the interval $[\alpha, \beta]$ to be (slightly) larger than the smallest interval containing all nodes. This can be bad because larger $[\alpha, \beta]$ will weaken the effectiveness of $S_{n, p^{\prime}}(\omega, \tau)$ in the later bounds on $\kappa_{p}\left(V_{\mathrm{c}}\right)$; for example $S_{n, p^{\prime}}(\omega, \tau)$ is decreasing in $\omega[10$.

\section{TWO UNIFORM BOUNDS}

We present two theorems here, one for any real $V_{\mathrm{c}}$ and one for $V_{\mathrm{c}}$ with nonnegative nodes. Their proofs will be given later after two lemmas. Again let $1 \leq p \leq \infty$ and $1 / p+1 / p^{\prime}=1$. 
Theorem 3.1. Under the conditions of Theorem 2.1, if

$$
k_{\max }-1 \leq \frac{n-1}{\sqrt{2}}\left[1-(1+\sqrt{2})^{-2 n+2}\right] \sim \frac{n-1}{\sqrt{2}},
$$

then

$$
\begin{aligned}
\kappa_{p}\left(V_{\mathrm{c}}\right) & \geq\left[\frac{\left(n-k_{\max }\right) !}{(n-1) !}\right]^{2} \frac{S_{n-1,1}(1,0)}{n^{1 / p^{\prime}}\lceil n / 2\rceil^{1 / p}} \\
& \sim\left[\frac{\left(n-k_{\max }\right) !}{(n-1) !}\right]^{2} \frac{[1+\sqrt{2}]^{n-1}}{n^{1 / p^{\prime}}\lceil n / 2\rceil^{1 / p}}
\end{aligned}
$$

Theorem 3.2. Under the conditions of Theorem 2.1, if all $\alpha_{i} \geq 0$ and

$$
k_{\max }-1 \leq \frac{n-1}{\sqrt{2}}\left[1-(1+\sqrt{2})^{-4(n-1)}\right]^{-1} \sim \frac{n-1}{\sqrt{2}},
$$

then

$$
\begin{aligned}
\kappa_{p}\left(V_{\mathrm{c}}\right) & \geq\left[\frac{\left(n-k_{\max }\right) !}{(n-1) !}\right]^{2} \frac{1}{2^{k_{\max }-1}} \frac{S_{n-1,1}(1 / 2,1)}{n} \\
& \sim\left[\frac{\left(n-k_{\max }\right) !}{(n-1) !}\right]^{2} \frac{1}{2^{k_{\max }-1}} \frac{[1+\sqrt{2}]^{2(n-1)}}{n} .
\end{aligned}
$$

Lemma 3.1. Let $j \geq 0$ and $m \geq 1$. $\rho^{j} S_{m, 1}(\rho, 0)$ is decreasing in $\rho$ for $0 \leq \rho \leq 1$ if

$$
j \leq \frac{m}{\sqrt{2}}\left[1-(1+\sqrt{2})^{-2 m}\right] \sim \frac{m}{\sqrt{2}} .
$$

Proof. We claim that under inequality (3.3), $\frac{d}{d \rho} \rho^{j} S_{m, 1}(\rho, 0) \leq 0$ for $0 \leq \rho \leq 1$. To this end, we notice that

$$
\frac{d}{d \rho} \rho^{j} S_{m, 1}(\rho, 0)=j \rho^{j-1} S_{m, 1}(\rho, 0)+\rho^{j} \frac{d}{d \rho} S_{m, 1}(\rho, 0) .
$$

Now for $0 \leq \rho \leq 1$ and by (2.8), we have

$$
\begin{aligned}
S_{m, 1}(\rho, 0) \leq & \frac{1}{2}\left[\frac{1}{\rho}+\sqrt{1+\frac{1}{\rho^{2}}}\right]^{m}\left[1+\epsilon^{-2 m}\right] \\
-\frac{d}{d \rho} S_{m, 1}(\rho, 0) \geq & \frac{m}{2}\left[\frac{1}{\rho}+\sqrt{1+\frac{1}{\rho^{2}}}\right]^{m-1}\left[1-\delta^{-2 m}\right] \\
& \times\left[\frac{1}{\rho^{2}}+\frac{1}{\rho^{2} \sqrt{1+\rho^{2}}}\right]
\end{aligned}
$$


where $\epsilon=1+\sqrt{2}$ and $\delta=0$ for even $m$, and $\epsilon=0$ and $\delta=1+\sqrt{2}$ for odd $m$. Therefore, for $\rho \leq 1$,

$$
\begin{aligned}
\frac{\frac{d}{d \rho} \rho^{j} S_{m, 1}(\rho, 0)}{m \rho^{j-1} S_{m, 1}(\rho, 0)} & =\frac{j}{m}+\frac{\rho \frac{d}{d \rho} S_{m, 1}(\rho, 0)}{m S_{m, 1}(\rho, 0)} \\
& \leq \frac{j}{m}-\frac{\rho\left[\frac{1}{\rho^{2}}+\frac{1}{\rho^{2} \sqrt{1+\rho^{2}}}\right]}{\frac{1}{\rho}+\sqrt{1+\frac{1}{\rho^{2}}}} \frac{1-\delta^{-2 m}}{1+\epsilon^{-2 m}} \\
& =\frac{j}{m}-\frac{1}{\sqrt{1+\rho^{2}}} \frac{1-\delta^{-2 m}}{1+\epsilon^{-2 m}} \\
& \leq \frac{j}{m}-\frac{1}{\sqrt{2}} \frac{1-\delta^{-2 m}}{1+\epsilon^{-2 m}} \\
& \leq \frac{j}{m}-\frac{1}{\sqrt{2}}\left[1-(1+\sqrt{2})^{-2 m}\right] \\
& \leq 0
\end{aligned}
$$

upon using (3.3).

Lemma 3.2. Let $j \geq 0, \gamma \geq 1$, and $m \geq 1$. For $j$ satisfying (3.3) and $\rho>0$,

$$
\rho^{j} \max \left\{\gamma, \rho^{m}\right\} S_{m, 1}(\rho, 0) \geq S_{m, 1}(1,0) .
$$

Proof. Let $\Phi_{1}=\rho^{j} \times \gamma S_{m, 1}(\rho, 0)$ and $\Phi_{2}=\rho^{j} \times \rho^{m} S_{m, 1}(\rho, 0)$. Then $\max \left\{\Phi_{1}, \Phi_{2}\right\}$ is $\Phi_{1}$ for $\rho \leq \gamma^{1 / m}$ and $\Phi_{2}$ for $\rho \geq \gamma^{1 / m}$. $\Phi_{2}$ is increasing in $\rho$ for $\rho>0$ because $\rho^{m} S_{m, 1}(\rho, 0)$ is a polynomial in $\rho$ with nonnegative coefficients and thus increasing in $\rho$ for $\rho>0$. So

$$
\max \left\{\Phi_{1}, \Phi_{2}\right\} \geq \Phi_{2} \geq S_{m, 1}(1,0) \text { for } \rho \geq 1 .
$$

For $0 \leq \rho \leq 1, \Phi_{1}$ is decreasing in $\rho$ by Lemma 3.1, and thus

$$
\max \left\{\Phi_{1}, \Phi_{2}\right\} \geq \Phi_{1} \geq S_{m, 1}(1,0) \text { for } \rho \leq 1 .
$$

This completes the proof.

Proof of Theorem 3.1. Setting $-\alpha=\beta=\alpha_{\max }$ in (2.5), we have, upon using (2.7),

$$
\begin{aligned}
\kappa_{p}\left(V_{\mathrm{c}}\right) & \geq \min _{1 \leq k \leq k_{\max }}\left[\frac{(n-k) !}{(n-1) !}\right]^{2} \alpha_{\max }^{k-1} \times \max \left\{\ell^{1 / p^{\prime}}, \alpha_{\max }^{n-1}\right\} \frac{S_{n-1,1}\left(\alpha_{\max }, 0\right)}{n^{1 / p^{\prime}}\lceil n / 2\rceil^{1 / p}} \\
& \geq\left[\frac{\left(n-k_{\max }\right) !}{(n-1) !}\right]^{2} \frac{1}{n^{1 / p^{\prime}}\lceil n / 2\rceil^{1 / p}} \min _{1 \leq k \leq k_{\max }} \widetilde{\Phi},
\end{aligned}
$$

where $\widetilde{\Phi}=\alpha_{\max }^{k-1} \times \max \left\{\ell^{1 / p^{\prime}}, \alpha_{\max }^{n-1}\right\} S_{n-1,1}\left(\alpha_{\max }, 0\right)$. Apply Lemma 3.2 with $j=$ $k-1, m=n-1, \gamma=\ell^{1 / p^{\prime}}$, and $\rho=\alpha_{\max }$ to get $\widetilde{\Phi} \geq S_{n-1,1}(1,0)$, as needed.

Proof of Theorem 3.2. Setting $0=\alpha<\beta=\alpha_{\max }$ in (2.5), we have, upon using (2.6),

$$
\begin{aligned}
\kappa_{p}\left(V_{\mathrm{c}}\right) & \geq \min _{1 \leq k \leq k_{\max }}\left[\frac{(n-k) !}{(n-1) !}\right]^{2}\left[\frac{\alpha_{\max }}{2}\right]^{k-1} \times \max \left\{\ell^{1 / p^{\prime}}, \alpha_{\max }^{n-1}\right\} \frac{S_{n-1,1}\left(\alpha_{\max } / 2,1\right)}{n} \\
& \geq\left[\frac{\left(n-k_{\max }\right) !}{(n-1) !}\right]^{2} \frac{1}{n 2^{k_{\max }-1}} \min _{1 \leq k \leq k_{\max }} \widetilde{\Psi},
\end{aligned}
$$


where

$$
\widetilde{\Psi}=\alpha_{\max }^{k-1} \times \max \left\{\ell^{1 / p^{\prime}}, \alpha_{\max }^{n-1}\right\} S_{n-1,1}\left(\alpha_{\max } / 2,1\right) .
$$

It can be verified by (2.3), (2.8), and (2.9) that

$$
S_{n-1,1}\left(\alpha_{\max } / 2,1\right)=S_{2(n-1), 1}\left(\sqrt{\alpha_{\max }}, 0\right) .
$$

Therefore

$$
\begin{aligned}
\widetilde{\Psi} & =\left(\sqrt{\alpha_{\max }}\right)^{2(k-1)} \times \max \left\{\ell^{1 / p^{\prime}},\left(\sqrt{\alpha_{\max }}\right)^{2(n-1)}\right\} S_{2(n-1), 1}\left(\sqrt{\alpha_{\max }}, 0\right) \\
& \geq S_{2(n-1), 1}(1,0),
\end{aligned}
$$

upon using Lemma 3.2 with $j=2(k-1), m=2(n-1), \gamma=\ell^{1 / p^{\prime}}$, and $\rho=\sqrt{\alpha_{\max }}$.

\section{Concluding Remarks}

We have obtained three lower bounds on the condition number $\kappa_{p}\left(V_{\mathrm{c}}\right)$ of a real confluent Vandermonde matrix $V_{\mathrm{c}}$. Two of them are uniform in the sense that they depend on $n$, the dimension of $V_{\mathrm{c}}$ only, while the other one is more general, as is the function of $n$ and $\alpha_{\max }$ and the interval $[\alpha, \beta]$ that contains all $\alpha_{j}$. These bounds grow exponentially for any fixed $k_{\max }$, much as expected. While it is not clear in general if (any of) our bounds are asymptotically optimal, in contrast to those for Vandermonde matrices by Beckermann [2] and recently by the author [10, our bounds are unlikely to be asymptotically optimal if $k_{\max }$ also grows, e.g., linearly in $n$. This is illustrated by the extreme example $k_{\max }=n$, as we commented in Section 1

We have focused on real confluent Vandermonde matrices here. It is conceivable that there would be much better conditioned complex confluent Vandermonde matrices or confluent Vandermonde-like matrices. This is partly an intuition one might get from that although real Vandermonde matrices are very ill-conditioned 17, 2, 10, 12, there exist very well-conditioned complex Vandermonde matrices and Vandermonde-like matrices [6, 11]. We plan to investigate this issue in future work.

\section{ACKNOWLEDGMEnT}

The author wishes to thank the anonymous referee for his constructive suggestions that improved and shortened the paper considerably.

\section{REFERENCES}

[1] George E. Andrews, Richard Askey, and Ranjan Roy, Special functions, Encyclopedia of Mathematics and its Applications, vol. 71, Cambridge University Press, Cambridge, UK, 1999. MR1688958 (2000g:33001)

[2] Bernhard Beckermann, The condition number of real Vandermonde, Krylov and positive definite Hankel matrices, Numer. Math. 85 (2000), no. 4, 553-577. MR.1771780(2001e:65075)

[3] Å. Björck and Victor Pereyra, Solution of Vandermonde systems of equations, Math. Comp. 24 (1970), no. 112, 893-903. MR0290541 (44:7721)

[4] Åke Björck and Tommy Elfving, Algorithms for confluent Vandermonde systems, Numer. Math. 21 (1973), 130-137. MR0336975 (49:1748)

[5] Peter Borwein and Tamás Erdélyi, Polynomials and polynomial inequalities, Graduate Texts in Mathematics, vol. 161, Springer, New York, 1995. MR.1367960 (97e:41001)

[6] Walter Gautschi, The condition of Vandermonde-like matrices involving orthogonal polynomials, Linear Algebra Appl. 52/53 (1983), 293-300. MR0709357(84i:65043) 
[7] _ How (un)stable are Vandermonde systems?, Asymptotic And Computational Analysis (R. Wong, ed.), Lecture Notes in Pure and Applied Mathematics, vol. 124, Marcel Dekker, Inc., New York and Basel, 1990, pp. 193-210. MR1052434 (91f:65080)

[8] N. J. Higham, Accuracy and stability of numerical algorithms, SIAM, Philadephia, 1996. MR.1368629 (97a:65047)

[9] Ren-Cang Li, Norms of certain matrices with applications to variations of the spectra of matrices and matrix pencils, Linear Algebra Appl. 182 (1993), 199-234. MR.1207083 (94c:15040)

[10] _ Asymptotically optimal lower bounds for the condition number of a real Vandermonde matrix, Technical Report 2004-05, Department of Mathematics, University of Kentucky, 2004, Avaliable at http://www.ms.uky.edu/ math/MAreport/. (Shortened version to appear in SIAM J. Matrix Appl.)

[11] Lothar Reichel and Gerhard Opfer, Chebyshev-Vandermonde systems, Math. Comp. 57 (1991), no. 196, 703-721. MR.1094957 (92a:65132)

[12] Evgenij E. Tyrtyshnikov, How bad are Hankel matrices?, Numer. Math. 67 (1994), 261-269. MR1262784 (94m:65075)

Department of Mathematics, University of Kentucky, Lexington, Kentucky 40506

E-mail address: rcli@ms.uky.edu

URL: http://www.ms. uky.edu/ ${ }^{\sim} \mathrm{rcli}$ 